\title{
Interactive biorobotics
}

Edoardo Datteri, RobotiCSS Lab, Department of Human Sciences for Education, University of Milano-Bicocca, edoardo.datteri@unimib.it

\begin{abstract}
What can interactive robots offer to the study of social behaviour? Philosophical reflections about the use of robotic models in animal research have focused so far on methods (including the so-called synthetic method) involving robots which do not interact with the target system. Yet, leading researchers have claimed that interactive robots may constitute powerful experimental tools to study collective behaviour. Can they live up to these epistemic expectations? This question is addressed here by focusing on a particular experimental methodology involving interactive robots which has been often adopted in animal research. This methodology is shown to differ from other robot-supported methods for the study of animal behaviour analysed in the philosophical literature, chiefly including the synthetic method. It is also discussed whether biomimicry (i.e., similarity between the robot and the target animal in behaviour, appearance, and internal mechanisms) and acceptability (i.e., whether or not the robot is accepted as a conspecific by the animal) are necessary for an interactive robot to be sensibly used in animal research according to this method.
\end{abstract}


(Accepted for publication in Synthese, 2020)

\section{Introduction}

Robots have been often called to play experimental roles for the study of animal and human behaviour. An early example is the implementation, in 1912, of Jacques Loeb's (1900) theory on the phototropism of moths in a robot whose light-seeking behaviour was later taken by Loeb himself as a support for his theory (Cordeschi, 2002). More recently, in so-called biorobotics, robotic systems have been used to study the behaviour of lobsters (Grasso et al., 2000), crickets (Reeve et al., 2005), portions of the human nervous system (Chou and Hannaford, 1997), and even extinct animals (Long, 2012). In these studies, one builds a robotic model of the target living system and draws theoretical conclusions regarding the latter from the analysis of the behaviour of the robot in controlled experimental settings. For example, Grasso and colleagues (2000) rejected a hypothesis on the mechanism enabling lobsters to track chemical streams in the water after attesting the inability of a robotic implementation of that mechanism to track chemical streams in an experimental pool.

Several methodological questions concerning this use of robots have been addressed in the philosophical literature (Cordeschi, 2002; Webb, 2001, 2006; Datteri, 2017; Datteri \& Tamburrini, 2007). Can robots provide genuine knowledge about the behaviour of living systems? What experimental procedures can be adopted in biorobotics? What kinds of theoretical results can be obtained? What are, if any, the advantages relative to different methodologies for obtaining the same results? These questions are of philosophical interest: philosophers of science have been traditionally concerned with the rational reconstruction of the methods adopted in scientific research and with the analysis 
(Accepted for publication in Synthese, 2020)

of their validity, and biorobotics provides several case studies to reflect on the roles that material models play in science.

Notably, methodological reflections about biorobotics have been chiefly focused, so far, on stand-alone robotic models which do not interact with the target living system. In the studies mentioned above, and in other biorobotics studies discussed in the philosophical literature, one observes the behaviour of the robotic model in isolation and draws theoretical conclusions about the target living system, with no interaction whatsoever between the robot and the target system. Recently, however, philosophers and scientists have occasionally claimed that interactive robots - i.e., robots able to interact with living systems - may play an important role in the study of animal and human behaviour. Krause and colleagues (2011, p. 369) claim that "interactive robots have the potential to revolutionise the study of social behaviour". A similar view is proposed by Griparić and colleagues (2017), who observe that "robots designed in such a way that they can generate particular stimuli and interact with animals, which consequently leads to acceptance of robots by animals as a part of their society, have shown a huge potential in animal behaviour research". Similar enthusiastic claims about the experimental value of interactive robots are made with reference to specific topics in animal research. For example, Marras and Porfiri (2012, p. 1856) "hypothesize that the integration of a fish-like robot within a group of live fish may enable fundamental research on collective animal behaviour and open new directions at the interface of robotics and marine biology".

These claims specifically concern non-human animals. However, it has been suggested that interactive robots may be used as experimental tools to study social 
behaviour in humans too. Dumouchel and Damiano (2017, pp. 53-54) claim that "introducing a robot in the company of human beings ... is an inherently social phenomenon that can be treated as a scientific experiment. Studying a whole range of reactions associated with acceptance and rejection ... and their short- and long-term effects cannot help but teach us many things about various aspects of human sociability". Brian Scassellati (2007; Scassellati et al., 2012) and Diehl and colleagues (2012) have claimed that robots may constitute useful tools to understand the causes of social and communicative impairments in people with autism.

It is important to pinpoint the claim made by these authors. That robots can exert transitory or even permanent influence on the behaviour of animals and humans is obvious. They can move, alter the environment in which other systems live, and produce visual, auditory, and tactile stimuli. Surrounding living systems are likely to be temporarily or permanently affected by their behaviour. What these authors are claiming is not the mere fact that robots may exert an influence on the surrounding living systems but that, by observing how living systems (humans and non-human animals) interact with robotic systems, one can acquire scientific knowledge about the determinants of collective behaviour. This is taken here to be the central tenet of so-called interactive biorobotics.

Interactive biorobotics gives rise to variants of the methodological questions raised above in connection with biorobotics. Can interactive robots provide genuine knowledge about the determinants of collective behaviour in living systems? What experimental procedures are adopted in interactive biorobotics? What kinds of theoretical results can be obtained? What are, if any, the advantages relative to different methodologies for obtaining 
the same results? These methodological questions have never been addressed in detail in the philosophical literature. Yet, they are of philosophical interest, as pointed out above in connection with biorobotics, as they concern the structure and the conditions of validity of model-based experimental strategies for the study of animal behaviour.

This article offers a methodological reflection on the experimental roles of interactive biorobots, thus filling a gap in the philosophical literature about the experimental roles of robots in the study of animal behaviour, which has been focused on non-interacting robots so far. More specifically, it has three goals.

The first goal, pursued in section 3, is to outline the structure of an interactive biorobotics methodology for the study of collective behaviour called here 'interactive stimulation methodology'. The methodology will be reconstructed based on the analysis of two studies in which interactive robots are used to theorize about fish schooling and collective decision making in groups of cockroaches. The description of the two studies, carried out in section 2, will be detailed enough to highlight the essential aspects of the methodology. Note that the purpose of this article is not to provide a comprehensive taxonomy of the experimental applications of interactive robots in the life sciences. There may be other and structurally different methods involving interactive robots for the study of animal and human behaviour. This article will focus on the interactive stimulation strategy only and offer an in-depth analysis of its structure. As pointed out later, however, many interactive stimulation studies have been carried out so far: reviews of the scientific literature have been offered by Krause and colleagues (2011) and Romano and colleagues (2019). For this reason, the proposed analysis may be a good starting point to initiate a 
(Accepted for publication in Synthese, 2020)

methodological reflection on the use of interactive robots as scientific tools in the life sciences.

The second goal, pursued in section 4 , is to argue that the interactive stimulation methodology differs in some key respects from the methodologies typically adopted in non-interactive biorobotics, including the so-called 'synthetic method' (Webb 2001, 2006; Cordeschi, 2002). Philosophical literature has chiefly focused on the synthetic method so far. This section therefore enables one to acknowledge that robots are being used in animal research in ways that have been unnoticed by the philosophical community. It also suggests that the analysis offered here fills a gap in the philosophical reflection on the use of robotic models in the life sciences.

The third goal, pursued in section 4, is to reflect about whether interactive robots, used according to the experimental procedure described in section 3, can provide genuine scientific knowledge about the determinants of collective behaviour in groups of living systems. This question will be addressed by identifying and discussing some auxiliary assumptions on which the validity of the method rests. These assumptions chiefly concern biomimicry and acceptability. Interactive bioroboticists often put a lot of effort into building robots that resemble as closely as possible, in appearance and behaviour, the animal(s) they interact with. Is this effort justified? Is robot biomimicry an essential requirement of "good" interactive stimulation studies? A separate discussion will be made about acceptability: should interacting robots be accepted as conspecifics by the animal(s) they interact with in the interactive stimulation methodology? It will be argued that the answer depends on the nature of the research question, even though additional 
methodological and conceptual issues must be addressed to fully understand the role of acceptability in interactive biorobotics. To prepare for a discussion of these questions, the following section describes in some detail two studies instantiating the interactive stimulation methodology.

\section{Self-organization in fish and cockroaches}

Fish aggregate in organized social groups called "shoals". This phenomenon, called "schooling", is the product of individual decisions based on a variety of sensory cues, whose contribution is yet to be fully determined for several species. The study reported by Polverino and colleagues (2013), called "fish study" here for short, focused on the phenomenon of schooling in golden shiners. The goal, as stated by the authors, was "to identify the determinants of attraction that regulate the collective behavior in social fish species when swimming together in a water tunnel" (p. 2). Specifically, the authors tested the hypothesis that individual golden shiners attract other golden shiners depending on two factors, namely their colour and their tail-beat frequency. This hypothesis was tested by observing if real-life golden shiners were attracted by robotic fish differing in the colour and tail-beat frequency.

More specifically, the authors built two robotic fish whose design "included visible fish anatomy, such as a dorsal fin, two pectoral fins, two pelvic fins, an anal fin, and a caudal fin" (p. 2). The two robots differed from one another in the colour: one was painted red, which is an unnatural colour for golden shiners, and the other one displayed the natural colour pattern of golden shiners - they were called the "red" and the "grey" robot respectively. In the experiments, either the red or the grey robot was fixed in a stationary 
position in the middle of a transparent water tunnel. ${ }^{1}$ Due to a particular actuation mechanism, the robots could flip the tail at a controlled frequency and reproduce the natural undulations observed in golden shiners. In each experimental session, a golden shiner was introduced in the water tunnel together with the robot. Two regions of the water tunnel were defined. One, called the "focal area", extending eight fish body lengths from the centre of mass of the robot, defined the interaction (or focal) zone: a golden shiner entering this area was considered to be interacting with the robot. The remaining part of the water tunnel was regarded as a non-interaction (or non-focal) zone. A total of eight experimental conditions were tried, differing from one another in the combination of colour (red or grey) and tail-beat frequency $(0,2,3$, and $4 \mathrm{~Hz})$ of the robot. In each condition, data on the position of the golden shiner with respect to the robot were collected during the session, in order to find out whether the golden shiner interacted with the robot (i.e., if it entered the interaction zone).

The results were as follows. When the red robot was used, no significant difference was detected in the time spent in the interaction and non-interaction zone, independently of the tail-beat frequency: "the time spent in both regions was not affected by the red robot tail-beat frequency" (p. 8). On the contrary, "the time spent in the focal region was affected by the grey robot tail-beat frequency" (p. 6). More specifically,

\footnotetext{
${ }^{1}$ Water tunnels are facilities for observing fish swimming behaviour: water flows in
} one direction at a controlled velocity, thus reducing relative positional changes of fish swimming in the opposite direction. 
fish spent significantly more time in the vicinity of the bioinspired robotic fish as its tail-beat frequency was set to $3 \mathrm{~Hz}(185.8 \mathrm{~s})$ as compared to the case when the robot tail-beat frequency was $0 \mathrm{~Hz}$ or $2 \mathrm{~Hz}(121.3 \mathrm{~s}$ or $132.7 \mathrm{~s}$, respectively). The time spent in the non-focal region of the test tank complemented the time spent in the focal region, that is, the mean time spent within the non-focal region was the lowest for a tail-beat frequency of $3 \mathrm{~Hz}$ (114.2 s). (p. 6)

To sum up. The goal of the authors of this study was "to investigate the interplay between visual and flow cues in the phenomenon of schooling in carangiform social fish" (p. 9), namely, to ascertain whether some aspects of the behaviour and appearance of individual golden shiners - colour and tail-beat frequency - affected distance between two individual fish, thus the formation of shoals. To achieve this goal, the authors systematically changed colour and tail-beat frequency in a robotic fish and observed the subsequent effects on the distance between the robot and a real-life golden shiner. The experimental results showed that, indeed, colour and tail-beat frequency make the difference, the distance between the two individuals reaching a minimum when a realistically painted robotic fish moving its tail at a frequency of $3 \mathrm{~Hz}$ is put in the water tunnel.

The second study discussed here concerns self-organization in communities of cockroaches. When put in an environment in which two dark shelters are present, groups of cockroaches eventually hide together under one of the two shelters only. A choice between the two is made by each insect, which is likely to be significantly influenced by the 
(Accepted for publication in Synthese, 2020)

behaviour of the other members of the group: this is a self-organization phenomenon. What aspects of the behaviour of individual cockroaches determine the choice of the shelter in this circumstance? This question, pointing to the identification of the individual factors modulating a particular kind of collective decision process, was addressed in the study reported by Halloy and colleagues (2007), called here the "cockroach study". Like the fish study, this question concerns the relationship between certain characteristics of individual living systems and the behaviour of the community. Like the fish study, this question was addressed by replacing some members of the community with robots, selectively altering their behaviour, and observing the effects of these alterations on the behaviour of the group.

The experimental environment was a circular arena with two shelters. The shelters consisted in Plexiglas discs, relatively distant from one another, and suspended by nylon threads over the surface of the arena. Red filters were placed on top of the discs: in some experimental conditions, one shelter had been made darker by putting more red filters relative to the other one. The experiments also involved a set of small robots called InsBots, which were similar in size to real-life cockroaches and programmed to mimic some aspects of the behaviour of real-life cockroaches. The behavioural rules were as follows. Each robot explored the environment randomly. Once it encountered a shelter, it rested in it for a time depending on two factors: 1) the darkness of the shelter (all the robots were initially configured to "prefer" darker shelters) and 2) the presence of other robots or cockroaches (they stayed longer in crowded shelters). They obviously had sensors and algorithms to detect obstacles, the presence of robots and cockroaches in the vicinity, and 
(Accepted for publication in Synthese, 2020)

the level of darkness of the shelter. The robots were also coated with a chemical substance releasing the characteristic odour of male cockroaches. Due to this substance, they were recognized as conspecifics by the living members of the group and able to influence their behaviour in some way.

The experiments were performed on a "pure" society comprising 16 living cockroaches and on a "mixed" society comprising 12 cockroaches and 4 InsBots. A first set of experiments was aimed to explore the dynamics of collective decision making in the "pure" and "mixed" society in the arena with two identically dark shelters. Consistently with the literature, in the "pure" case all the cockroaches aggregated under the same shelter. Interestingly, the same phenomenon was observed in the "mixed" case too: "in 28 of 30 trials (93\%) mixed groups presented a clear choice for one of the shelters, and $75 \%$ of cockroaches and $85 \%$ of robots aggregated under the same shelter" (p. 1157). A second set of experiments was aimed to assess collective decision making with one shelter being darker than the other one. Experiments with the pure group resulted in a collective preference for the darker shelter: "when cockroach groups selected one of the shelters (22 of 30 trials), the darker shelter was selected in $73 \%$ of the cases and the lighter one in only $27 \%$ of the cases" (p. 1157).

Notably, in the experiments with the mixed group, the InsBots were programmed to prefer light shelters instead of dark shelters. This behavioural alteration was found to affect the behaviour of the group to a significant degree: "the shelter less preferred by the cockroaches (i.e., the lighter one) was selected by mixed groups in $61 \%$ of the trials, versus only $27 \%$ of the trials done without robots" (p. 1157). Hiding under the lighter shelter, 
(Accepted for publication in Synthese, 2020)

when a darker one is available, is an unnatural choice for real-life cockroaches. However, changing individual preferences in the InsBots led the group to make this choice, suggesting that individual preference for darker (lighter) shelters is a determinant of collective aggregation under the darker (lighter) shelter. This result had another interesting implication. The lighter shelter, in this condition, was selected by the mixed group in $61 \%$ of the trials, meaning that in the remaining $39 \%$ of the trials the mixed group - including the InsBots programmed to prefer lighter shelters - aggregated under the darker shelter. In some trials, re-programmed robotic cockroaches led the group to make an unnatural choice for real-life cockroaches, while in other trials unaltered real-life cockroaches induced the re-programmed robots to make a choice that was "unnatural" for them. In the authors' words, "in some trials the choice was induced by the robots, and in others by the cockroaches. The robots did not act as a mere attractant but were integrated into the decision-making process of the society" (p. 1157).

To sum up. The goal of the study was to find out what aspects of individual behaviour influence collective selection of one shelter out of two in a group of cockroaches. To address this question, the authors replaced some members of the group with robotic cockroaches, selectively intervened on some aspects of their behaviour namely, preference for darker shelters - and observed the effects on the behaviour of the group. This intervention was found to significantly alter the behaviour of the group, which in some cases ended up making a biologically unnatural decision, suggesting that individual preference for darker (lighter) shelters is a determinant of collective selection of the darker (lighter) shelter. 


\section{The structure of the interactive stimulation methodology}

The fish and cockroach studies resemble one another in a number of methodological respects. More specifically, the research questions addressed in the two studies share a common structure, and there are some interesting commonalities in the experimental procedures too. As far as the research question is concerned, note that the two studies involved robots interacting with living systems in a suitably controlled environment. In both cases, the authors manipulated some aspects of the behaviour and appearance of the robots and observed the resulting effects in the behaviour of the mixed group composed of the $\operatorname{robot}(\mathrm{s})$ and the target living system(s). However, the authors were not interested in observing animal reactions to robotic behaviours per se: their ultimate goal was to learn something about the determinants of fish and cockroach collective behaviour. The fish study aimed to identify the effect of colour and tail-beat frequency variations on mutual attraction between golden shiners. The goal of the cockroach study was to identify the determinants of collective decision making in groups of cockroaches. The two studies addressed research questions concerning the determinants of the collective behaviour in pure communities of living systems, even though their methodology involved observation of mixed communities composed of robots and living systems.

The structure of the research questions addressed in these studies can be more precisely schematized as follows. In both cases, the system under investigation included a group of living systems situated in an experimental environment and was characterized in terms of some parameters. Some of them - e.g., tail-beat frequency or individual preference for darker or lighter shelters - concerned the physical appearance and behaviour 
(Accepted for publication in Synthese, 2020)

of individual members of the group. Other parameters - e g., the distance between two individual fish or the number of cockroaches under the shelters - represented features of the group. Letters I (for "individual") and C (for "collective") will be used from now on to denote these sets of parameters. The structure of the research questions addressed in the two studies can be schematized accordingly as follows:

What relationship holds between the value of parameters $I=\left\{i_{1}, \ldots, i_{m}\right\}$ and the value of parameters $C=\left\{c_{1}, \ldots, c_{n}\right\}$ under boundary conditions $B=\left\{b_{1}\right.$, $\left.\ldots, b_{\mathrm{p}}\right\} ?$

I and $\mathrm{C}$ will be called I-parameters and C-parameters respectively. The set B includes parameters (called B-parameters) describing boundary (e.g., environmental) conditions supposed to have a non-negligible influence on the relationship between I-parameters and C-parameters. In the fish study, the I-parameters included colour and tail-beat frequency, the C-parameters included distance between two individual fish, and the B-parameters characterized the structure of the experimental setting (e.g., the structure of the water tunnel). Using the experimental setting described above, the authors managed to obtain information about the relationship holding between colour and tail-beat frequency, on the one hand, and the distance between two golden shiners on the other hand. In the cockroach study, the I-parameters included the degree of individual preference for darker shelters, the C-parameters included the number of cockroaches under the two shelters, and the Bparameters characterized the structure of the environment (e.g., its being circular with two shelters which may be identically dark or not). The authors gained information on whether 
(Accepted for publication in Synthese, 2020)

individual preference for darker (lighter) shelters may alter the number of cockroaches under the darker (shelter).

There are some interesting commonalities in the experimental procedures adopted in the two studies to address these questions. Indeed, in both cases, the authors replaced some members of the group with robotic systems, thus forming a mixed community of fish and cockroaches. Then, they selectively intervened on some parameters of the robotic replacer(s) - called here $I_{R}$-parameters, "R" for "robotic" - and observed changes in the behaviour of the mixed community - represented in terms of $\mathrm{C}_{\mathrm{M}}$-parameters, " $\mathrm{M}$ " for "mixed". Observing how the values of the $\mathrm{C}_{\mathrm{M}}$-parameters changed after interventions on the $\mathrm{I}_{\mathrm{R}}$-parameters, the authors drew conclusions about the relationship between the Iparameters and the C-parameters. More specifically, the goal of the fish study was to ascertain the relationship between colour and tail-beat frequency (I) and distance between two fish (C). The authors replaced one fish with a robot, altered its colour and tail-beat frequency $\left(I_{R}\right)$, and observed the effects on the distance between fish and robot $\left(C_{M}\right)$. The results were brought to bear on the relationship between I and C. Similarly, the goal of the cockroach study was to ascertain the relationship between individual preference for darker or lighter shelters (I) and collective selection of one shelter (C). The authors replaced some cockroach with InsBots, altered their preference $\left(I_{R}\right)$, observed the number of individuals under the shelters $\left(\mathrm{C}_{\mathrm{M}}\right)$, and brought the results to bear on the relationship between I and $\mathrm{C}$.

To sum up, the fish and cockroach studies resemble each other in the structure of the research question and in the experimental procedure. They are regarded here as instances of the interactive stimulation methodology, which has been reconstructed here in 
(Accepted for publication in Synthese, 2020)

pursuance of the first goal of this article. As stressed before, it is not claimed here that the interactive stimulation methodology is the only possible methodology involving interactive robots in animal research. However, several additional examples can be found in the recent literature on the behaviour of honeybees (Griparić et al., 2017), fish (Landgraf et al., 2016; Romano et al., 2017), and chickens (Gribovskiy et al., 2010): for a state of the art, see (Krause et al., 2011; Romano et al., 2019).

One may legitimately ask what advantages this strategy offers relative to the more canonical practice of directly intervening on a living member of the group. The advantages are practical and ethical. Directly intervening on I-parameters - e.g., controlling the tailbeat frequency and the colour of living golden shiners, or altering the preference of living cockroaches - may be technically difficult or impossible. Some forms of direct intervention on living systems may also be ethically questionable. One may also ask whether non-robotic devices could be used as replacers in this method, allowing one to avoid the technical complexities involved in building a robot. Indeed, computer animations have been used to address topics such as mating preferences in animals (Künzler and Bakker, 1998). In computer animations, one may easily alter the morphology of the virtual animal and observe how collective behaviour changes (Rosenthal and Evans, 1998). However, as pointed out by Krause and colleagues (2011), interaction with virtual animals is restricted to visual stimuli in two dimensions. To study the relationship between tail-beat frequency and shoal formation, and the relationship between individual hiding preferences and collective decision making, a system able to flap its tail and hide under shelters is needed. 


\section{Interactive biorobotics and the synthetic method}

Philosophers of science and philosophically minded scientist have often discussed about the role of robotic models in animal research (Floreano et al., 2014; Pfeifer and Bongard, 2006; Pfeifer and Scheier, 1999; Webb, 2000, 2001, 2006; Cordeschi, 2002; Datteri and Tamburrini, 2007; Datteri, 2017). However, this literature almost exclusively focuses on non-interactive biorobotics. Non-interactive biorobotic studies involve robotic systems which implement a theoretical model of the behaviour of a living system: by observing how the robot behaves in controlled experimental settings, one acquires new knowledge about the target system. Two varieties of this methodology can be found in the literature, none of which, as discussed below, conforms to the interactive stimulation methodology.

The vast majority of non-interactive biorobotics studies adopts the so-called synthetic method, SM from now on (Cordeschi, 2002). The purpose of the SM is to test a how-possibly theoretical model of the mechanism ${ }^{2}$ enabling a living system to behave in a

${ }^{2}$ The terms "mechanism" and "mechanistic model" are used in this article in the sense clarified by the vast contemporary literature on mechanistic modelling and explanation (see Glennan \& Illari, 2018 for an up-to-date discussion). No further analysis of these concepts is made here, as this article is concerned neither with mechanistic modelling and explanation nor with the role of robots in testing mechanistic models or explanations (a role which, according to some authors, e.g., Cordeschi, 2002 and Craver, 2010, is occasionally assigned to robots and hybrid systems in neuroscience and animal research). 
(Accepted for publication in Synthese, 2020)

certain way. The model is implemented in the robot, and the behaviour of the robot is compared with the behaviour of the target system in suitable experimental settings. Assuming that the robot accurately implements the theoretical model, matches and mismatches between the two can be taken as empirical basis to corroborate or reject it. Several examples of this method can be found in the scientific literature. In the study described by Grasso and colleagues (2000), an underwater robotic system called RoboLobster was built which implemented a hypothesis on the mechanism enabling lobsters to track the source of chemical streams in the water. In the experiments, the robot failed to hit the source in many cases, leading the authors to conclude that the hypothesis was too simplistic. Reeve and colleagues (2005) built a robot implementing a theoretical model of cricket phonotaxis which, unlike the lobster study, succeeded in matching the sound-localization abilities of real-life crickets, leading the authors to corroborate the hypothesis. Other examples are discussed by Datteri (2017). To sum up, the synthetic method has two distinctive features.

- SM1: the goal is to test a how-possibly model of the mechanism enabling the target system to behave in a certain way (note that the theoretical model under scrutiny is the model implemented in the machine).

- SM2: the experimental procedure crucially involves comparing the behaviour of the robot with the behaviour of the biological living system whose theoretical model is implemented in the machine. The result of this comparison is brought to bear on the plausibility of the theoretical model under scrutiny. 
While the goal of the SM is to test a hypothesis on how the target system produces the observed behaviour, other non-interactive biorobotic studies aim to acquire knowledge about what behaviour the target system would produce in certain circumstances. In this case, one implements a theoretical model whose predictive validity - unlike the "synthetic" case - is taken for granted at the beginning of the study. Then, the behaviour of the robot is interpreted as the behaviour that the target system would produce in the same circumstances (under the assumption that the robot accurately implements the theoretical model). A case in point is a study carried out by John Long and colleagues at Vassar College, New York. They built a robot, called Madeleine, reproducing some known features of the morphology and swimming mechanisms of aquatic tetrapods. The purpose was not to discover the mechanism enabling tetrapods to swim, but to obtain data on the swimming performances of tetrapods - in particular, of an extinct animal called the Plesiosaurus - under some conditions (Long, 2012; Long et al., 2006). In other words, the goal was to gain novel information on the behaviour of the target system, and not to test a theoretical model of that behaviour, using a robotic model of it: this strategy is particularly useful when the behaviour of the target system is hard or impossible to observe using alternative means (e.g., because the target system is extinct, as in the case of the Plesiosaurus). Since the term "prediction" is occasionally used in the philosophy of science community to refer to the generation of novel data on a system, though not necessarily concerning its future states, this class of studies will be called predictionoriented (PO) here. Prediction-oriented studies have some distinctive features. 
(Accepted for publication in Synthese, 2020)

- PO1: the goal is to predict the behaviour of the living system whose theoretical model is implemented in the robot.

- PO2: the behaviour of the robotic system in particular circumstances is regarded as informative about the behaviour that the target system would display in similar circumstances. $^{3}$

In the cockroach and fish studies, like in SM and PO studies, theoretical conclusions about living systems are drawn from experiments involving robots. But the analogy ends here: the two studies do not follow the synthetic method and cannot be classified as prediction-oriented either. As a general remark, note that in SM and PO studies new knowledge about the target living system - concerning the plausibility of the implemented theoretical model and the behaviour that the target system would produce in particular circumstances, respectively - is inferred from the analysis of the behaviour of a stand-alone robot. It is by observing whether RoboLobster performs efficient chemotaxis that Grasso and colleagues drew conclusions on the plausibility of the implemented

${ }^{3}$ The SM and PO strategies sketched here are akin to explanatory and predictive strategies involving non-robotic, computer simulations of biological and physical phenomena discussed by Weisberg (2013) and Winsberg (2010). A detailed analysis of the SM and PO is beyond the scope of this article (see Datteri, 2017 for a more thorough discussion): reference to these strategies is made here only to emphasize the peculiarity of the interactive stimulation strategy relative to more traditional uses of robots in animal research. 
(Accepted for publication in Synthese, 2020)

mechanistic model qua model of lobster chemotaxis. It is by observing Madeleine's behaviour that John Long and colleagues inferred conclusions on the behaviour of the Plesiosaurus. On the contrary, theoretical conclusions about the collective behaviour of cockroaches and fish shoals are not drawn, in the two studies analysed here, by observing the behaviour of the robots, but by observing how other living systems reacted to the presence of the robot.

More specifically, the cockroach study fails to display the two characteristic features of the synthetic method discussed above. As far as SM1 is concerned, the goal of the study is not to test a how-possibly theoretical model of cockroach behaviour, i.e., to test the model implemented in the machine, as is the case with the synthetic method. The goal, as stated before, is rather to identify the factors modulating the behaviour of the community. As far as SM2 is concerned, the experimental procedure does not involve any comparison between the behaviour of the InsBots and the behaviour of the living systems whose theoretical model is implemented in the machine (i.e., of real-life cockroaches). The cockroach study is not a predictive-oriented study either. As far as PO1 is concerned, the goal is not to predict the behaviour of individual cockroaches but to identify the factors modulating the behaviour of the community. As far as PO2 is concerned, the behaviour of the InsBots is not regarded as informative about the behaviour that the target system would display in similar circumstances. The authors never inferred the future behaviour of individual cockroaches from the actual behaviour of the InsBots (recall that the goal of the study is not to predict the behaviour of cockroaches). 
Along the same lines, it can be argued that the fish study does not follow the synthetic method: the purpose of the study is not to test a theoretical model of the swimming behaviour of individual golden shiners, and no comparison is made between the behaviour of the robot and of golden shiners. It is not a predictive-oriented study either, as the robotic fish is not used to predict the behaviour of golden shiners, and the behaviour of the robotic fish is not regarded as informative about the behaviour that actual golden shines would produce in the same circumstances.

To sum up. The methodology adopted in the cockroach and fish studies does not display some distinctive features of the synthetic method and of prediction-oriented studies, which constitute typical forms of non-interactive biorobotics. More generally, in interactive stimulation studies, theoretical conclusions about the target living species are not obtained by observing how the robotic system behaves, as in traditional non-interactive biorobotics, but by observing how insects and animals react to the presence of the robot. They therefore significantly differ, from a methodological point of view, from the experimental strategies on which philosophical reflections about the role of robotic models in the life sciences have focused so far.

\section{Biomimicry, acceptability, and background features of the robot}

\subsection{Auxiliary assumptions: from hybrid to pure communities}

Can interactive robots provide genuine knowledge about the determinants of collective behaviour in pure communities of living systems? This question is addressed here in connection with the interactive stimulation methodology. In this methodology, theoretical conclusions about the relationship between I and $\mathrm{C}$ are inferred from results concerning the 
relationship between $I_{R}$ and $C_{M}$. In other words, changes in $C_{M}$ (i.e., in the behaviour of the hybrid community) resulting from changes of $I_{R}$ (i.e., of features of the robots) are taken to be informative about changes in $\mathrm{C}$ (i.e., in the behaviour of a non-hybrid community group) that would result from the corresponding changes of I (i.e., of features of the nonreplaced living systems). In the fish study, changes in fish-robot distance resulting from interventions on the colour and tail-beat frequency of the robot are taken to be informative about changes in fish-fish distance that would result from interventions on the colour and tail-beat frequency of one of the two fish. Similarly, in the cockroach study, changes in the collective behaviour of the mixed community resulting from interventions on robots' preference for darker shelters are regarded as informative about the collective behaviour that a pure community would display after altering the preference of some members of the group. To safely infer theoretical conclusions about the I-C relationship from results concerning the $I_{R}-C_{M}$ relationship, one needs auxiliary assumptions concerning 1) the relationship between $I_{R}$ and I, and 2) the impact of features of the robotic replacer(s) which are not represented in $I_{R}$ - called here the background features of the robot - on the behaviour of the mixed community.

First, there must be a close correspondence between I and $I_{R}$. If the purpose is to ascertain whether colour and tail-beat frequency affects distance between fish, colour and tail-beat frequency is what must be changed in the robot. The specific values assigned to the $I_{R}$-parameters in the experiments matter too: if the purpose is to ascertain whether distance between fish decreases when the fish displays a realistic pigmentation, the robot must display a realistic pigmentation. Otherwise, the reaction of the fish cannot be 
(Accepted for publication in Synthese, 2020)

informative about the reaction that the same fish would display when interacting with a living golden shiner with realistic pigmentation.

Second, to bring results on the $\mathrm{I}_{\mathrm{R}}-\mathrm{C}_{\mathrm{M}}$ relationship to bear on the I-C relationship, one must neutralize potential disturbances introduced by background features of the robot. The $I_{R}$-parameters in the fish study included colour and tail-beat frequency of the robot. The robotic fish had many other characteristics which were not represented in that set, including two rods anchoring the robotic fish to the water tunnel. One rod was used to keep the trunk fixed, and the other one connected an Arduino-controlled servomotor to the mobile parts of the fish in order to produce tail flipping. The two rods were found to cause vorticity patterns in the upper area of the tunnel. In principle, these turbulences might produce an artefactual disturbance: the reaction of the living fish to the robot, in that part of the tunnel, might be different from the reaction that the same fish would have produced to a living (rod-less) fish in the very same conditions, preventing the authors from generalizing from robot-fish interaction to fish-fish interaction. This problem was neutralized by ignoring distance data acquired when the fish was in the upper area of the tunnel.

\subsection{Biomimicry}

The need to tackle disturbances caused by background features of the robot is interestingly connected to the effort put by the authors of the fish and cockroach studies in building biomimetic robots. Note that, as pointed out in section 4, the robots involved in noninteractive biorobotic studies implement theoretical models of the target biological system. In the synthetic method, the robot accurately implements a how-possibly theoretical model 
of the behaviour under investigation. In prediction-oriented studies, the robot implements a predictively valid model of the target behaviour. As discussed before, interactive stimulation studies are not prediction-oriented and do not conform to the synthetic method either. Still, some physical and behavioural features of the robots involved in the two studies mimic some aspects of their biological counterparts.

As explicitly pointed out by Polverino and colleagues (2013), the engineering design of the robotic fish "was bioinspired to mimic the aspect ratio, body shape, size, and species-specific locomotion pattern observed in the golden shiner" (p. 2). The authors aimed to test the hypothesis that "a bioinspired robotic fish is able to elicit attraction in a live fish as a consequence of the visual and flow cues it offers" (p. 2). "The robotic fish was designed to mimic the locomotion of golden shiners and match their morphology" (p. 8). Notably, the authors aimed "to mimic the swimming motion of live golden shiners. Biomimicry elements in the experiments were incorporated into the design phase and also assessed from a hydrodynamic standpoint" (p. 3). Indeed, "to assess the degree of biomimicry" (p. 3), the authors compared the undulations of the robotic fish with classical models of carangiform swimming and with experimental data on the undulation patterns of golden shiners. This comparative analysis was specifically meant "to validate the ability of the robotic fish to reproduce carangiform swimming" (p. 4).

Similarly, the physical and algorithmic structure of the InsBots was accurately shaped taking the physical structure and behaviour of real-life cockroaches into account. In particular, the authors attempted to mimic cockroaches in terms of size, motion and behaviour ("robots have to move and react to cockroaches and other robots like a real 
(Accepted for publication in Synthese, 2020)

cockroach among its group. Their movements are designed to avoid excessive bumping into insects and display similar speed and movement as insects"), and environment perception abilities ("robots have to distinguish between walls or obstacles and shelters of different darkness"), see Halloy and colleagues (2007b, p. 2).

Why is biological mimicry sought for in these studies? One might answer by arguing that a robot must be physically and behaviourally similar to a living system to influence its behaviour. This is false, however: a robot which is physically and behaviourally different from a fish (or cockroach) may affect its behaviour to a great degree, possibly by triggering escape reactions. This answer also fails to pinpoint the reason why biological mimicry was sought for in the two studies. Indeed, their goal was not, strictly speaking, to influence the behaviour of fish and cockroaches, but rather to find out whether collective behaviour was affected in certain ways by the individual characteristics of the robot. The discovery that no collective alteration was produced in certain circumstances - e.g., that the robot-fish distance was not affected by changes in the red robot's tail-beat frequency - was a scientifically interesting result indeed. Thus, even if it was true that a robot must be physically and behaviourally similar to a living system to influence its behaviour, this would not explain the authors' pursuit of biomimicry, as the goal of the studies was not to influence the behaviour of fish and cockroaches.

A more plausible answer is that a certain level of biomimicry is required to bring results on the $I_{R}-C_{M}$ relationship to bear on the I-C relationship. Two robots were used in the fish study, which were both approximately of the same size of golden shiners. The choice of a bioinspired size - size being a background feature of the robot - was justified 
(Accepted for publication in Synthese, 2020)

by pointing out that an unnatural size might have introduced disturbances difficult to neutralize. Indeed, interactive stimulation experiments involving alterations of tail-beat frequency in a robot had been carried out by Marras and Porfiri (2012). However, while the findings obtained in that study "have contributed to validating the hypothesis that the hydrodynamic return offered by a robotic fish is a determinant for robotic fish's attractiveness to live fish, the robot used therein was considerably larger than live fish. The unmatched size between live and robotic fish in the study by Marras and Porfiri (2012) may act as a confound for elucidating the role of flow cues produced by fish locomotion on collective behaviour" (p. 2). The choice of a bioinspired size in the fish study was justified by pointing out that an unnatural size might have prevented the authors to draw conclusions on the behaviour of the "pure" community from observation of the behaviour of the "mixed" community - in other word, to safely bring results on the $\mathrm{I}_{R}-\mathrm{C}_{\mathrm{M}}$ relationship to bear on the I-C relationship. The choice of InsBots having bioinspired size and speed may be justified along similar lines. To sum up, biomimicry is not sought for in these studies to exert a stronger influence on real-life fish and cockroaches, but to neutralize potential disturbances introduced by (biologically unrealistic) background features of the robot.

\subsection{Acceptability}

Odour is one of the biologically inspired characteristics of the robots involved in the cockroach study. As pointed out before, the InsBots were coated with a special chemical substance releasing the characteristic odour of male cockroaches. Due to their odour, the InsBots were accepted as conspecifics by the community of cockroaches and were able to 
(Accepted for publication in Synthese, 2020)

participate in collective decision making with the rest of the group. Note that acceptance of the InsBots in the community was required to bring the behaviour of the "mixed" group to bear on the behaviour of the "pure" group. If the InsBots had not been accepted in the community, the behaviour of the rest of the group would have been probably influenced by their presence nonetheless, possibly in the form of an escape reaction. However, it is reasonable to believe that the collective behavioural effects produced by changing individual InsBots preference for darker shelters would not have been informative about the collective behavioural effects deriving from similar changes in real-life cockroaches. Indeed, non-accepted InsBots would have acted as foreign bodies in the community and would not have participated in collective selection of a shelter. The goal of the study is to understand if individual preference for darker or lighter shelters has some effect on the collective selection of a shelter, and to achieve this goal one has to intervene on individual preference in agents participating in collective shelter selection: this is why the authors put a great deal of effort in inducing the cockroaches to accept the robots as part of the community. Non-accepted robots would have been "bad" replacers for an interactive biorobotics study concerning collective decision making. The first experimental part of the cockroach study, showing that in "normal" conditions the collective behaviour of the "mixed" group is similar to the collective behaviour of the "pure" group (in both cases all the members aggregated under one of the shelters), can be interpreted as an experimental test of acceptance: the InsBots contribute to collective decision making in a way that is very similar to the way real-life cockroaches contribute to the same decision-making process. 
(Accepted for publication in Synthese, 2020)

In the cockroach study, acceptance (of the robots by the living members of the group) is therefore essential to bring results on the $\mathrm{I}_{\mathrm{R}}-\mathrm{C}_{\mathrm{M}}$ relationship to bear on the I-C relationship. Is acceptance essential in interactive stimulation studies generally? More explicitly, must a robot be accepted as a conspecific by the living system(s) it interacts with in this methodology? This claim is made by Landgraf and colleagues (2016): "a key prerequisite for the use of robots in the study of animal behavior is to develop systems towards which real animals react relatively naturally [...]. The robot should be accepted as a conspecific by live animals".

From the analysis of the cockroach study carried out here, one may conclude that acceptance is crucial when the goal is to inquire into the factors affecting the behaviour of a community which is engaged in collective decision making. Note, however, that the interactive stimulation strategy can be adopted to achieve different goals - for example, to identify the conditions under which a group of individuals will participate in collective decision making. The fish study is a case in point. The goal was to isolate the conditions under which two fish interact with one another - which can be paraphrased as the goal to isolate the conditions under which one golden shiner accepts another golden shiner as a part of the shoal, thus maintaining a short distance from it. Acceptance was the dependent variable under investigation, and, for this reason, it could not have been treated as a prerequisite: guaranteeing acceptance in every experimental session would have rendered the overall methodology totally incoherent with the character of the research question. On the contrary, the dependent variable in the cockroach study was the number of individuals 
(Accepted for publication in Synthese, 2020)

under the two shelters in a group which was assumed to participate in a collective decisionmaking process: this is why acceptance was a key prerequisite in that study.

To sum up. The analysis of the cockroach study carried out here provides some reasons to believe that the robot should be accepted as a conspecific by the community if the goal is to identify the individual determinants of collective decision making. However, acceptance is not required if the goal is to identify the conditions under which a group of individuals will participate in a collective decision-making process. Taken together, these two considerations enable one to conclude that whether acceptance is required or not in this methodology, is a question that depends on the nature of the research question. Note that this provisional conclusion is based on the analysis of two case studies concerning the behaviour of non-human animals. A full understanding of the role of acceptance in interactive biorobotics, possibly encompassing studies concerning human social cognition, requires one to address further methodological questions that are formulated in the next section and left to future research.

\section{Summary and concluding remarks}

Robotic technologies are pervading society and playing important roles in an increasing number of everyday activities, often by actively engaging with humans and animals in collaborative decision-making processes. What can interactive robots offer to the study of animal behaviour? This broad question has been addressed here with a methodological analysis of an experimental strategy involving interactive robots called interactive stimulation strategy. It consists in replacing some members of a group with robotic systems, selectively altering certain features of the robotic replacers, and observing the 
(Accepted for publication in Synthese, 2020)

effects at the group level. The goal is to identify the individual determinants of collective behaviour. This strategy has been shown to differ, from a methodological point of view, from more traditional non-interactive biorobotic studies. Can it provide genuine knowledge about collective behaviour in animal groups? This question has been addressed by focusing on some background assumptions needed to infer theoretical conclusions concerning collective behaviour of "pure" groups from experimental results concerning "hybrid" groups composed of animals and robots. They chiefly concern biomimicry and acceptability. Forms of biomimicry are often sought for in non-interactive biorobotics, and acceptability has been claimed to be essential to use interactive robots in animal research. It has been argued that, while a certain degree of biomimicry may be important to neutralize potential disturbances introduced by background features of the robot, acceptance is not always required in robot-supported interactive stimulation studies. In other terms, a robot which is not accepted as part of a society may nevertheless influence its behaviour in ways that are interesting for the study of collective behaviour.

Philosophical literature on the epistemic role of robotic systems in cognitive, behavioural, and neural science has mainly focused on the synthetic method. It has been occasionally suggested that observing the dynamics of animal-robot interaction may offer valuable insights for the study of human and animal behaviour. However, no in-depth analysis of specific interaction-based experimental strategies and of the involved methodological complexities had been carried out so far. This article attempts to initiate a methodological discussion of the experimental value of animal-robot interaction, thus 
(Accepted for publication in Synthese, 2020)

contributing to unravelling the many roles that robots can play as scientific instruments in contemporary cognitive and neuroscience.

As pointed out in the Introduction, this article was not intended to provide a comprehensive taxonomy of the potential applications of interactive robots to the study of animal and human behaviour. The goal was rather to identify and discuss one specific interactive biorobotics methodology at some level of detail. The analysis carried out here can be enriched and complemented by identifying other methodologies involving interactive robots, and by addressing various methodological and conceptual questions arising from the claims made here.

First, other experimental methods involving human-robot or animal-robot interaction may be identified which differ in some respects from the method analysed here. For example, in so-called cognetics (Rognini and Blanke, 2016), robots are used to automatically deliver stimuli triggering particular cognitive or emotional states in human beings, such as the feeling of somebody being nearby, to understand the neural correlates of illusions (Blanke et al., 2014). The strategy adopted in these studies does not seem to conform to the strategy discussed here, as the robot does not replace any member of a group. Whether there is a fundamental difference, from a methodological point of view, between cognetics and the interactive stimulation strategy, is a question to be addressed in future research.

Second, some claims made here give rise to further methodological and conceptual questions. For example, acceptability and its role in the interactive stimulation method 
(Accepted for publication in Synthese, 2020)

have been discussed in section 5.3 with reference to two case studies concerning cockroach and fish behaviour only. A more extensive analysis of acceptability, possibly considering other case studies, is needed to fully understand its role in interactive biorobotics. First, what is to be accepted by a community of living systems? In the cockroach study, acceptance seemingly equates to the ability to participate in a collective decision-making process. But acceptance may be defined in different ways. A living system may recognize a robot as a conspecific (according to some sense of "recognizing") without letting it participate in a collective decision-making process. Conversely, a living system may accept to collaborate with a robot in a decision-making process without recognizing it as a conspecific. There may be different degrees and types of acceptance (see for example de Graaf and Ben Allouch, 2013, for an analysis of some dimensions of acceptance in humanrobot interaction). Second, how to assess whether a robot has been accepted by a group of living systems? One may compare the outcome of a collective decision-making process in "pure" and "mixed" groups, as in the cockroach study. However, in some cases and with some living species, comparing the outcome of a complicated decision-making process may not be enough to evaluate acceptance - one may need to compare the dynamics of the process itself, according to some criteria. Or, one may carry out tests which do not involve comparisons between "pure" and "mixed" groups. The choice of the best evaluation method clearly depends on how one interprets the concept of "acceptance". Third, how to obtain high degrees of acceptance? In the cockroach study, acceptance is obtained by ensuring a certain level of physical and behavioural biomimicry. However, this strategy may not work in other cases and with other living species: an animal may accept to 
(Accepted for publication in Synthese, 2020)

collaborate with a robot because its behaviour is functional to reaching a certain goal, regardless of the degree of biological plausibility of the robot (see Romano et al. 2019 for a reflection on the factors determining animal perception of robots as conspecifics). These questions are left to future research: the goal of this article was to lay some groundwork for the analysis of the many roles that interactive robots, and robots generally, can play in the study of animal and human behaviour.

Acknowledgement. The author thanks two anonymous referees for their insightful comments and acknowledges with gratitude the suggestions received at the 2019 Conference of the European Philosophy Association (Geneva), at the 2018 Conference of the Italian Association of Cognitive Science (Genova), and during a seminar held at the Center for Logic, Language and Cognition of the University of Torino in 2018, where previous versions of this paper were presented.

\section{References}

Blanke, O., Pozeg, P., Hara, M., Heydrich, L., Serino, A., Yamamoto, A., ... Rognini, G. (2014). Neurological and robot-controlled induction of an apparition. Current Biology, 24(22), 2681-2686. https://doi.org/10.1016/j.cub.2014.09.049

Chou, C.-P., \& Hannaford, B. (1997). Study of human forearm posture maintenance with a physiologically based robotic arm and spinal level neural controller. Biological Cybernetics, 76(4), 285-298. https://doi.org/10.1007/s004220050340 
(Accepted for publication in Synthese, 2020)

Cordeschi, R. (2002). The Discovery of the Artificial. Behavior, Mind and Machines Before and Beyond Cybernetics. Dordrecht: Springer Netherlands. https://doi.org/10.1007/978-94-015-9870-5

Craver, C. F. (2010). Prosthetic Models. Philosophy of Science, 77(December), 840-851. https://doi.org/10.1086/656822

Datteri, E. (2017). Biorobotics. In L. Magnani \& T. W. Bertolotti (Eds.), Springer Handbook of Model-Based Science (pp. 817-837). Heildelberg/Berlin: Springer.

Datteri, E., \& Tamburrini, G. (2007). Biorobotic Experiments for the Discovery of Biological Mechanisms. Philosophy of Science, 74(3), 409-430.

de Graaf, M. M. A., \& Ben Allouch, S. (2013). Exploring influencing variables for the acceptance of social robots. Robotics and Autonomous Systems, 61(12), 1476-1486.

Diehl, J. J., Schmitt, L. M., Villano, M., \& Crowell, C. R. (2012). The clinical use of robots for individuals with Autism Spectrum Disorders: A critical review. Research in Autism Spectrum Disorders, 6(1), 249-262. https://doi.org/10.1016/j.rasd.2011.05.006

Dumouchel, P., \& Damiano, L. (2017). Living with Robots. Cambridge, MA: Harvard University Press.

Floreano, D., Ijspeert, A. J., \& Schaal, S. (2014). Robotics and neuroscience. Current Biology, 24(18), R910-R920. https://doi.org/10.1016/j.cub.2014.07.058

Glennan, S., \& Illari, P. (Eds.). (2018). The Routledge Handbook of Mechanisms and Mechanical Philosophy. Routledge, New York. 
(Accepted for publication in Synthese, 2020)

Grasso, F. W., Consi, T. R., Mountain, D. C., \& Atema, J. (2000). Biomimetic robot lobster performs chemo-orientation in turbulence using a pair of spatially separated sensors: Progress and challenges. Robotics and Autonomous Systems, 30(1-2), 115131. https://doi.org/10.1016/S0921-8890(99)00068-8

Gribovskiy, A., Halloy, J., Deneubourg, J. L., Bleuler, H., \& Mondada, F. (2010). Towards mixed societies of chickens and robots. IEEE/RSJ 2010 International Conference on Intelligent Robots and Systems, IROS 2010 - Conference Proceedings, 4722-4728. https://doi.org/10.1109/IROS.2010.5649542

Griparić, K., Haus, T., Miklić, D., Polić, M., \& Bogdan, S. (2017). A robotic system for researching social integration in honeybees. PloS One, 12(8), e0181977. https://doi.org/10.1371/journal.pone.0181977

Halloy, J., Sempo, G., Caprari, G., Rivault, C., Asadpour, M., Tache, F., ... Deneubourg, J. L. (2007). Social integration of robots into groups of cockroaches to control selforganized choices (Supporting Material). Science, 318(5853), 1155-1158. https://doi.org/10.1126/science.1144259

Halloy, J., Sempo, G., Caprari, G., Rivault, C., Asadpour, M., Tâche, F., ... Deneubourg, J. L. (2007). Social integration of robots into groups of cockroaches to control selforganized choices. Science, 318(5853), 1155-1158. https://doi.org/10.1126/science.1144259

Krause, J., Winfield, A. F. T., \& Deneubourg, J. L. (2011). Interactive robots in experimental biology. Trends in Ecology and Evolution, 26(7), 369-375. 
(Accepted for publication in Synthese, 2020)

https://doi.org/10.1016/j.tree.2011.03.015

Künzler, R., \& Bakker, T. C. M. (1998). Computer Animations as a Tool in the Study of Mating Preferences. Behaviour, 135(8/9), 1137-1159.

https://doi.org/10.1163/156853998792913537

Landgraf, T., Bierbach, D., Nguyen, H., Muggelberg, N., Romanczuk, P., \& Krause, J. (2016). RoboFish: increased acceptance of interactive robotic fish with realistic eyes and natural motion patterns by live Trinidadian guppies. Bioinspiration \& Biomimetics, 11(1), 015001. https://doi.org/10.1088/1748-3190/11/1/015001

Loeb, J. (1900). Comparative physiology of the brain and comparative psychology. New York: G.P. Putman’s Sons. https://doi.org/10.5962/bhl.title.1896

Long, J. (2012). Darwin's Devices. What Evolving Robots Can Teach Us About the History of Life and the Future of Technology. New York: Basic Books.

Long, J. H., Schumacher, J., Livingston, N., \& Kemp, M. (2006). Four flippers or two? Tetrapodal swimming with an aquatic robot. Bioinspiration \& Biomimetics, 1(1), 2029. https://doi.org/10.1088/1748-3182/1/1/003

Marras, S., \& Porfiri, M. (2012). Fish and robots swimming together : attraction towards the robot demands biomimetic locomotion. Journal of the Royal Society Interface, 9(February), 1856-1868.

Pfeifer, R., \& Bongard, J. (2006). How the Body Shapes the Way We Think. A New View of Intelligence. Cambridge, MA: The MIT Press. 
(Accepted for publication in Synthese, 2020)

Pfeifer, R., \& Scheier, C. (1999). Understanding Intelligence. Cambridge, MA: The MIT Press.

Polverino, G., Phamduy, P., \& Porfiri, M. (2013). Fish and Robots Swimming Together in a Water Tunnel: Robot Color and Tail-Beat Frequency Influence Fish Behavior. PLoS ONE, 8(10), 47-50. https://doi.org/10.1371/journal.pone.0077589

Reeve, R., Webb, B., Horchler, A., Indiveri, G., \& Quinn, R. (2005). New technologies for testing a model of cricket phonotaxis on an outdoor robot. Robotics and Autonomous Systems, 51(1), 41-54. https://doi.org/10.1016/j.robot.2004.08.010

Rognini, G., \& Blanke, O. (2016). Cognetics: Robotic Interfaces for the Conscious Mind. Trends in Cognitive Sciences, 20(3), 162-164.

https://doi.org/10.1016/j.tics.2015.12.002

Romano, D., Benelli, G., Donati, E., Remorini, D., Canale, A., \& Stefanini, C. (2017). Multiple cues produced by a robotic fish modulate aggressive behaviour in Siamese fighting fishes. Scientific Reports, 7(1), 1-11. https://doi.org/10.1038/s41598-01704840-0

Rosenthal, G. G., \& Evans, C. S. (1998). Female preference for swords in Xiphophorus helleri reflects a bias for large apparent size. Proceedings of the National Academy of Science USA, 95(April), 4431-4436. https://doi.org/10.1073/pnas.95.8.4431

Scassellati, B. (2007). "How Social Robots Will Help Us to Diagnose, Treat, and Understand Autism”. In S. Thrun, R. Brooks, H. Durrant-Whyte (eds), Robotics Research, STAR 28, 552-563. 
(Accepted for publication in Synthese, 2020)

Scassellati, B., Admoni, H., \& Matarić, M. (2012). Robots for use in autism research. Annual Review of Biomedical Engineering, 14, 275-294. https://doi.org/10.1146/annurev-bioeng-071811-150036

Webb, B. (2000). What does robotics offer animal behaviour? Animal Behaviour, 60(5), 545-558. https://doi.org/10.1006/anbe.2000.1514

Webb, B. (2001). Can robots make good models of biological behaviour? The Behavioral and Brain Sciences, 24(6), 1033-1050. Retrieved from http://www.ncbi.nlm.nih.gov/pubmed/12412325

Webb, Barbara. (2006). Validating biorobotic models. Journal of Neural Engineering, 3, R25-R35. https://doi.org/10.1088/1741-2560/3/3/R01

Weisberg, M. (2013). Simulation and Similarity. Using Models to Understand the World. Oxford: Oxford University Press.

Winsberg, E. B. (2010). Science in the Age of Computer Simulation. Chicago and London: University of Chicago Press. 